\title{
Revisão taxonômica de Acianthera sect. Pleurobotryae (Orchidaceae, Pleurothallidinae) ${ }^{1}$
}

\author{
Vinícius Trettel Rodrigues ${ }^{2,5}$, Eric de Camargo Smidt ${ }^{3}$ e Fábio de Barros ${ }^{4}$
}

Recebido: 29.08.2015; aceito: 9.09.2015

\begin{abstract}
Taxonomic revision of Acianthera sect. Pleurobotryae (Orchidaceae, Pleurothallidinae)). Acianthera Scheidew. comprises about 200 species with Neotropical distribution; in the most recent proposal of taxonomic synopsis for the genus, Chiron \& van den Berg created 10 sections, among which Acianthera sect. Pleurobotryae, subject of this study. The section Pleurobotryae can be distinguished from other Acianthera sections by the long unguiculate lip with a mobile articulation, and the cylindrical to laterally flattened leaves, attached to a thread like cauloma. Their specimens are rather distributed in Southern and Southeastern Brazil and Northeastern Argentina. In this work a taxonomic study of the section Pleurobotryae is presented. Three new synonyms and a new combination are proposed for the group. Of the six species previously assigned to this section, only four are accepted here: Acianthera atropurpurea (Barb.Rodr.) Chiron \& van den Berg, A. crepiniana (Cogn.) Chiron \& van den Berg, A. hatschbachii (Schltr.) Chiron \& van den Berg, and A. mantiquyrana (Barb.Rodr.) V.T. Rodrigues \& F. Barros.
\end{abstract}

Keywords: Atlantic forest, Epidendroideae, Pleurobotryum, Pleurothallis

RESUMO - (Revisão taxonômica de Acianthera sect. Pleurobotryae (Orchidaceae, Pleurothallidinae)). Acianthera Scheidew. envolve cerca de 200 espécies de distribuição Neotropical; na mais recente sinopse taxonômica proposta para o gênero, Chiron \& van den Berg criaram dez seções, dentre elas a Acianthera sect. Pleurobotryae, objeto deste estudo. A seção Pleurobotryae distingue-se das demais seções de Acianthera por possuir flores com labelo de articulação móvel, longamente unguiculado, aliado às folhas teretiformes ou lateralmente compressas, unidas a um cauloma filiforme; seus representantes encontram-se distribuidos preferencialmente no Sul e Sudeste do Brasil e Nordeste da Argentina. Neste trabalho é apresentada a revisão taxonômica da seção Pleurobotryae na qual são propostas três novas sinonímias e uma nova combinação. Das seis espécies anteriormente atribuídas a esta seção, somente quatro são aqui aceitas: Acianthera atropurpurea (Barb.Rodr.) Chiron \& van den Berg, A. crepiniana (Cogn.) Chiron \& van den Berg, A. hatschbachii (Schltr.) Chiron \& van den Berg e A. mantiquyrana (Barb. Rodr.) V.T. Rodrigues \& F. Barros.

Palavras-chave: Epidendroideae, Floresta Atlântica, Pleurobotryum, Pleurothallis

\section{Introdução}

O gênero Acianthera Scheidw. foi restabelecido por Pridgeon \& Chase (2001) dada a constatação do polifiletismo de Pleurothallis R.Br. s.l. (Pridgeon et al. 2001). Engloba cerca de 200 espécies (Pridgeon et al. 2005), dentre as quais 104 são endêmicas do Brasil (Barros et al. 2015). Na mais recente sinopse do gênero, Chiron \& van den Berg (2012) apresentaram um novo sistema de classificação para Acianthera subdividindo-o em diversas seções e subseções, dentre elas Acianthera sect. Pleurobotryae Chiron \& van den Berg, que inclui as espécies tradicionalmente alocadas no gênero Pleurobotryum Barb.Rodr. Essa proposta teve como base uma hipótese filogenética para Pleurothallis s.l. apresentada por Chiron et al. (2012).

Acianthera é amplamente distribuído por toda a América tropical e subtropical, mas as espécies da seção Pleurobotryae estão restritas ao Sul e Sudeste do Brasil, com duas espécies alcançando o Norte da Argentina.

1. Parte da Tese de Doutorado do primeiro Autor

2. Universidade Braz Cubas, Av. Francisco Rodrigues Filho 1233, Vila Mogilar, 08773-380 Mogi das Cruzes, SP, Brasil

3. Universidade Federal do Paraná, Centro Politécnico, Setor de Ciências Biológicas, Departamento de Botânica, Caixa Postal 19031, 81531-990 Curitiba, PR, Brasil

4. Instituto de Botânica, Núcleo de Pesquisa Orquidário do Estado, Av. Miguel Estéfano 3687, 04301-902 São Paulo, SP, Brasil

5. Autor para correspondência: rodriguesvt@yahoo.com.br 
Ao propor o gênero Pleurobotryum, BarbosaRodrigues (1877) afirmou que este apresentava grande afinidade com o que, na época, entendiase como gênero Pleurothallis, tanto pelo número de polínias (2), como pela posição da antera (incumbente), mas distinguia-se facilmente pelas flores com labelo de articulação móvel e pelo hábito das plantas muito diferente, fazendo alusão às folhas teretiformes. Cogniaux (1893-1896) reduziu o status do gênero à categoria de seção, como Pleurothallis sect. Pleurobotryum (Barb.Rodr.) Cogn. e, sem ter ciência de que o epíteto "atropurpurea" já estava ocupado por Pleurothallis atropurpurea (Lindl.) Lindl. [= Zootrophion atropurpureum (Lindl.) Luer] descrita por Lindley (1842), transferiu a espécie de Barbosa Rodrigues para o gênero Pleurothallis, com o nome Pleurothallis atropurpurea (Barb.Rodr.) Cogn., criando, assim, um nome ilegítimo. Cogniaux (1893-1896) também incluiu nessa seção Pleurothallis teretifolia Rolfe, espécie descrita pouco antes por Rolfe (1892), cujo epíteto faz referência às folhas cilíndricas do exemplar por ele examinado. Na mesma obra, foi descrita outra espécie, Pleurothallis crepiniana Cogn., originalmente posicionada em Pleurothallis sect. Sarcodanthae Cogn., mas posteriormente acomodada em Pleurobotryum por Hoehne (1936).

O gênero Pleurobotryum foi restabelecido por Hoehne (1936) com o acréscimo de Pleurobotryum hatschbachii (Schltr.) Hoehne (= Pleurothallis hatschbachii Schltr.), e para tanto o autor justificou:"Indubitavelmente as duas plantas pertencem a este gênero criado por Barbosa Rodrigues, cujo característico mais evidente não é o labello unguiculado, mas sim o porte brassavoliforme da planta..."(sic.). Ainda no mesmo artigo, Hoehne (1936) apresentou uma chave de identificação para algumas Pleurothallidineae que apresentam labelo unguiculado e teceu os seguintes comentários acerca delas: "No caso que quizessemos agrupar as espécies referidas por Schlechter, pela forma do labelo unguiculado, teríamos que incluir as seguintes espécies: Pleurothallis mantiquyrana Barb.Rodr., P. rhabdosepala Schtr., P. crepiniana Cogn. e $P$. unguiculata Hoehne.... Mas é claro que estas não são todas as espécies que apresentam limbo largo e mais ou menos oblongo ou orbicular, sostido por uma parte liguliforme, bem longa. Para inclui-las no gênero Pleurobotryum que aqui restabelecemos com as duas espécies: P. atropurpureum Barb.Rodr. e P. hatschbachii Schtlr., seria necessário, portanto que se procurasse separar todas para que com os seus caractereres erigir um genero com outra diagnose em que estes fossem respeitados."(sic.).

Embora fique claro que o restabelecimento do gênero Pleurobotryum proposto por Hoehne (1936) tenha visado à circunscrição de apenas duas espécies (Pleurobotryum atropurpureum Barb.Rodr. e $P$. hatschbachii Schtlr.) o autor apresentou uma chave de identificação na qual as novas combinações são feitas ampliando a circunscrição do gênero independentemente da intenção e demais considerações do autor. Fato que contradiz o artigo 34.1 do Código Internacional de Nomenclatura Botânica que afirma: "Um nome não é validamente publicado quando não é aceito pelo autor na publicação original...".

Após a adição de novas espécies em Pleurobotryum proposta por Hoehne (1936) outras espécies foram alocadas nesse gênero por outros autores. Brade \& Pabst (1952) transferiram Pleurothallis albopurpurea Kraenzl. para Pleurobotryum e Garay (1952), certamente sem ter conhecimento do trabalho de Brade \& Pabst (1952), também propôs a mesma transferência, meses depois. Pabst (1975) transferiu Pleurothallis subulifolia Kraenzl. para o gênero e excluiu dele P. unguiculatum (Hoehne) Hoehne, sinonimizando-o com Pleurothallis octophrys Rchb.f.

A partir de 1978, Carlyle August Luer, deu início a uma série de publicações que apresentam uma proposta sistemática para Pleurothallidinae, na qual dividiu Pleurothallis em diversos subgêneros, seções e subseções, algumas destas similares aos grupos informais tratados por Pabst \& Dungs (1975). Luer (1986) propôs que Pleurobotryum fosse considerado subgênero de Pleurothallis, aceitando sete das oito espécies propostas para o gênero até então, à excessão de Pleurothallis octophrys Kraenzl. (sinônimo de Pleurothallis unguiculata Hoehne) que Luer (1986) considerou membro de Pleurothallis subgen. Acianthera sect. Tomentosae Luer. Desde então, Luer vem publicando novos tratamentos taxonômicos baseados na morfologia e/ou combinando a morfologia às mais recentes propostas filogenéticas para muitos desses grupos.

A partir de 2004 Luer vem elevando à categoria de gênero todos os subgêneros de Pleurothallis criados anteriormente por ele próprio, bem como restabelecendo todos aqueles que havia subordinado a gêneros anteriormente existentes (e.g. Luer 2004, 2006).

Chiron \& Van den Berg (2012), ao proporem a inclusão de Pleurobotryum em Acianthera, consideraram a seção composta por sete espécies.

Este trabalho teve por objetivo a revisão taxonômica de Acianthera sect. Pleurobotryae, 
e inclui uma chave de identificação, dados de distribuição geográfica das espécies e ilustrações, assim como discussão de aspectos taxonômicos.

\section{Material e métodos}

O trabalho teve início com o levantamento da coleção de exsicatas depositadas nos herbários $\mathrm{BAB}$, BHCB, HB, HCF, MBM, R, RB, SI, SP,UEC e UPCB, além da análise de fotografias de materiais-tipo disponíveis na internet, depositados nos herbários: BR, HBG, K e S (acrônimos de acordo com Thiers 2013).

A padronização morfológica das descrições seguiu os trabalhos de Radford et al. (1974) para estruturas em geral e de Dressler (1981) para estruturas específicas da família Orchidaceae.

$\mathrm{Na}$ ilustração das espécies foram utilizadas, eventualmente, fotografias de outros autores, objetivando ilustrar os espécimes que não apresentaram flores no período deste trabalho; nestes casos, os créditos foram indicados por meio das seguintes legendas nas próprias fotos: L.F.V.: Luiz Filipe Varella; D.H.B.: Dalton Holland Baptista; F.V.: Francisney Vinhos.

\section{Resultados e Discussão}

Dasnove espécies atribuídas aogênero Pleurobotryum ou relacionadas a ele, hoje parcialmente alocadas em Aciantera sect. Pleurobotryae [Pleurobotryum atropurpureum Barb.Rodr., P. mantiquyranum (Barb. Rodr.) Hoehne, P. rhabdosepalum (Schltr.) Hoehne, P. subulifolium (Kraenzl.) Pabst, P. crepinianum (Cogn.) Hoehne, P. albopurpureum (Kraenzl.) Garay, $P$. hatschbachii (Schltr.) Hoehne, P. unguiculatum (Hoehne) Hoehne e Pleurothallis teretifolia Rolfe] apenas quatro podem ser claramente diferenciadas sendo os demais nomes sinônimos de espécies já conhecidas, ou não pertencem a esta seção, como é o caso de P. unguiculatum. O levantamento dos exemplares nos herbários consultados mostra que essa seção não é endêmica do Brasil como apontado anteriormente por diversos autores (Pabst \& Dungs 1975, Barros et al. 2009, 2013), bem como amplia o conhecimento sobre sobre sua distribuição no território nacional. A escassez de materiais depositados nos herbários sugere que as espécies do gênero são raras na natureza e maiores estudos devem ser feitos a fim de averiguar seu real status de conservação, uma vez que nenhuma delas está relacionada em listas de espécies vegetais ameaçadas e, no entanto, entre todos os materiais examinados apenas um exemplar foi coletado no século XXI.
Acianthera sect. Pleurobotryae Chiron \& van den Berg $\equiv$ Pleurobotryum Barb.Rodr., Gen. Spec. Orchid. 1: 20. 1877 E Pleurothallis sect. Pleurobotryum (Barb.Rodr.) Cogn. in C.F.P. von Martius \& auct. suc. (eds.), Fl. Bras. 3(4): 588. $1896 \equiv$ Pleurothallis subgen. Pleurobotryum (Barb.Rodr.) Luer, Monogr. Syst. Bot. Missouri Bot. Gard.20: 53.1986. Tipo: Acianthera atropurpurea (Barb.Rodr.) Chiron \& van den Berg

Plantas epífitas, pendentes ou eretas. Cauloma capilar, articulado. Folhas cilíndricas ou lateralmente compressas. Inflorescência em racemo. Sépalas laterais com base adnata ao pé do ginostêmio; labelo longamente unguiculado, articulado ao pé do ginostêmio; clinândrio com margem ciliada; antera apical, unilocular; cavidade estigmática ventral; polínias 2, piriformes, lateralmente compressas.

Chave de identificação para as espécies de Acianthera sect. Pleurobotryae

1. Folhas lateralmente compressas ...... 2. A. crepiniana 1. Folhas cilíndricas

2. Plantas eretas, com até $12 \mathrm{~cm}$ alt.; flores amarelas a alaranjadas estriadas de castanho-vinoso 4. A. mantiquyrana

2. Plantas pendentes, com mais de $12 \mathrm{~cm}$ alt.; flores predominantemente verdes, castanhas ou vinosas

3. Flores verdes ou castanhas pontilhadas de vinoso; sépalas suberetas com ápice tenuemente reflexo; sépala dorsal aderente às sépalas laterais ..... 3. A. hatschbachii

3. Flores vinosas; sépalas eretas com ápice tenuemente inflexo; sépala dorsal livre das sépalas laterais .......... 1. A. atropurpurea

1. Acianthera atropurpurea (Barb.Rodr.) Chiron \& van den Berg, Richardiana 12(2): 72. $2012 \equiv$ Pleurobotryum atropurpureum Barb.Rodr., Gen. Spec. Orchid. 1: 27. 1877 E Pleurothallis atropurpurea (Barb.Rodr.) Cogn. in C.F.P. von Martius \& auct. suc. (eds.), Fl. Bras. 3(4): 588. 1896. Tipo: BRASIL. Minas Gerais: Poços de Caldas, J. Barbosa Rodrigues s.n. (holótipo RB, perdido; lectótipo aqui designado: tab. 154 do vol. 3 da Iconographie des Orchidées du Brésil, depositada em RB e reproduzida por Sprunger (1996, p. 201). 
$=$ Pleurothallis teretifolia Rolfe, Gard. Chron., ser. 3, 12: 521. 1892. Tipo: BRASIL. Pernambuco (?): Caldas, Charlesworth s.n., X-1982 (holótipo K000294042, foto K!).

Figuras 1 a, $3 \mathrm{c}$

Planta epífita, pendente, cespitosa, 25-34 cm alt. Rizoma cilíndrico, articulado, ca. $9 \mathrm{~mm}$ compr., $2 \mathrm{~mm}$ diâm. Cauloma 8,2-13,1 × ca. 0,2 cm, pendente, ligeiramente arqueado, 4-5-articulado, parcialmente revestido por 4-5 bainhas tubulosas que se fragmentam na maturidade. Folha $8,4-11,4 \times 0,1-0,3 \mathrm{~cm}$, verdeescura, ereta ou subereta, cilíndrica, coriácea, séssil, ápice agudo, base atenuada. Inflorescência arqueada, 3-6 flores sucessivas, ca. $6 \mathrm{~cm}$ compr.; pedúnculo ca. 1,6 cm compr., filiforme, bráctea floral ca. $3 \mathrm{~mm}$ compr., estramínea, tubulosa, ápice obliquamente truncado. Flores vinosas; pedicelo ca. $2 \mathrm{~mm}$ compr., filiforme; ovário ca. $2 \mathrm{~mm}$ compr., articulado ao pedicelo, piriforme, pubescente, 6-costado; sépala dorsal 9-10 × 3-4 mm, carnosa, ereta, estreitamente elíptica, 3-nervada, pubescente, aderente às sépalas laterais quase até o ápice, ápice agudo, tenuemente inflexo; sépalas laterais coalescentes na base até 1/3 do comprimento, carnosas, eretas, estreitamente elípticas, 3-nervadas, pubescentes, ápice agudo, inflexo; pétalas ca. $4 \times 1 \mathrm{~mm}$, carnosas, eretas, espatuladas, 1-nervadas, ápice apiculado; labelo longamente unguiculado, ca. $5 \times 2 \mathrm{~mm}$, vinoso, membranáceo, subereto, unguículo ca. $2 \mathrm{~mm}$ compr., base 2-auriculada, lâmina ca. $3 \times 2 \mathrm{~mm}$ larg., estreitamente elíptica, 3-nervada, ápice arredondado, base truncada, margem inteira. Ginostêmio ca. 4 mm compr., subclaviforme, delgado, encurvado, pubescente.

Material examinado: BRASIL. Minas Gerais: Caldas, 20-IV-1929, A.J. Sampaio s.n. (R20344); Carangola, 16-III-1997, L.S. Leoni \& A. Toscano de Brito 3653 (HB); Caxambú, 22-IV-1948, E. Dantas 8 (RB); Oliveira, 5-VI-2003, E. Tameirão Neto 3579 (BHCB); Paraisópolis, 23-IV-1927, F.C. Hoehne s.n. (SP19167); São Tomé das Letras, VII-1950, A.C. Brade 20590 (RB). São PAULO: Botucatu, 29-I-1973, A. Amaral Jr. 782 (SP); Campos do Jordão, X-1937, P.C. Porto 3399 (RB).

Acianthera atropurpurea é a única espécie da sect. Pleurobotryae que apresenta flores purpúreas que não se abrem completamente. Embora seja uma espécie de fácil identificação e de pouca variação na morfologia floral e no colorido de suas flores, alguns equívocos na identificação desta espécie foram encontrados.
Correa (1975) cita a espécie para a Argentina, porém o exame da descrição bem como dos materiais citados pela autora, mostra que as amostras pertencem a A. hatschbachii (Schltr.) Chiron \& van den Berg. Embora a autora faça, posteriormente, a retificação deste equívoco (Correa 1975), o erro se perpetuou até os dias de hoje como observado na identificação de alguns materiais depositados nos herbários da Argentina. Outro provável equívoco ocorre com P. teretifolia Rolfe, em que o autor indica o Estado de Pernambuco, município de Caldas, local que com esta designação, não foi localizado, porém há materiais coletados por A.J. Sampaio no município de Caldas no estado de Minas Gerais. Como nenhum outro material foi encontrado com indicação de coleta no Estado de Pernambuco, esta localização foi descartada da distribuição geográfica da espécie.

A espécie ocorre preferencialmente em regiões serranas de Floresta Atlântica no Sudoeste e Sul do Estado de Minas Gerais e Nordeste e Centro Sul do Estado de São Paulo entre 950 e 1.600 m de altitude (figura 4).

2. Acianthera crepiniana (Cogn.) Chiron \& van den Berg, Richardiana 12(2): 73. $2012 \equiv$ Pleurothallis crepiniana Cogn., in C.F.P. von Martius \& auct. suc.(eds.), Fl. Bras. 3(4): 542. 1896 $\equiv$ Pleurobotryum crepinianum (Cogn.) Hoehne, Bol. Mus. Nac. Rio de Janeiro 12(2): 28. 1936. Tipo: BRASIL. MinaS Gerais: Caldas, Ribeirão dos Bugres, Lindberg 516 (holótipo BR6585013, foto BR!).

= Pleurothallis albopurpurea Kraenzl., Ark. Bot. 16(8): 11. 1920. $\equiv$ Pleurobotryum albopurpureum (Kraenzl.) Brade \& Pabst, Orquídea (Rio de Janeiro) 14: 75.1952 (março-abril / 1952) Eleurobotryum albopurpureum (Kraenzl.) Garay, Arch. Jard. Bot. Rio de Janeiro 12: 170. 1952 (dezembro/1952), nom. superfl. $\equiv$ Acianthera albopurpurea (Kraenzl.) Chiron \& van den Berg, Richardiana 12(2): 73. 2012. Tipo: Lectótipo, aqui designado: BRASIL. Paraná: Serrinha, Dusén 8516. (S-R 4948, foto S!; isolectótipos S 05-6339, HBG500467, HBG501874, fotos HBG!, K000584051, foto K!), synon. nov.

Figuras 1 b, c, d; 3 a, b

Planta epífita, ereta, cespitosa, 5,5-15 cm alt. Rizoma cilíndrico, articulado, ca. $1,1 \times 0,2 \mathrm{~cm}$. Cauloma 2,5-8,8 $\times$ ca. $0,1 \mathrm{~cm}$, ereto ou subereto, ligeiramente arqueado, 3-4-articulado, totalmente revestido por 3-4 bainhas tubulosas que se fragmentam na maturidade. Folha 2,7-6,5 × 0,5-0,9 cm, verde- 
escura, ereta ou subereta, lateralmente compressa, carnosa, séssil, sulcada na base, ápice agudo, base atenuada. Inflorescência ereta, 5-10 flores simultâneas; pedúnculo 6-9 $\mathrm{mm}$ compr., filiforme, bráctea floral ca. $3 \mathrm{~mm}$ compr., estramínea, tubulosa, ápice obliquamente truncado. Flores rosadas, verdes ou castanhas, estriadas ou pontilhadas de vinoso; pedicelo ca. $1 \mathrm{~mm}$ compr., filiforme; ovário ca. $3 \mathrm{~mm}$ compr., articulado ao pedicelo, obcônico, pubérulo, 6-costado; sépala dorsal 5-7 $\times$ ca. $2 \mathrm{~mm}$, carnosa, subpatente, estreitamente elíptica, 3-nervada, pubescente na face externa, ápice agudo, nervuras vinosas; sépalas laterais coalescentes por todo o comprimento, carnosas, subpatentes, estreitamente elípticas, 3-nervadas, pubescentes na face externa, ápice agudo, nervuras vinosas; pétalas ca. $4 \times 1 \mathrm{~mm}$, membranáceas, eretas, estreitamente espatuladas, 1-nervadas, ápice agudo, nervuras vinosas; labelo longamente unguiculado, ca. $4 \times 2 \mathrm{~mm}$ larg., vinoso, carnoso, subereto, unguículo ca. $2 \mathrm{~mm}$ compr., base provida de duas projeções triangulares, lâmina ca. $2 \times 2 \mathrm{~mm}$, lunulada, 2-carenada, ápice arredondado, margem denticulada.
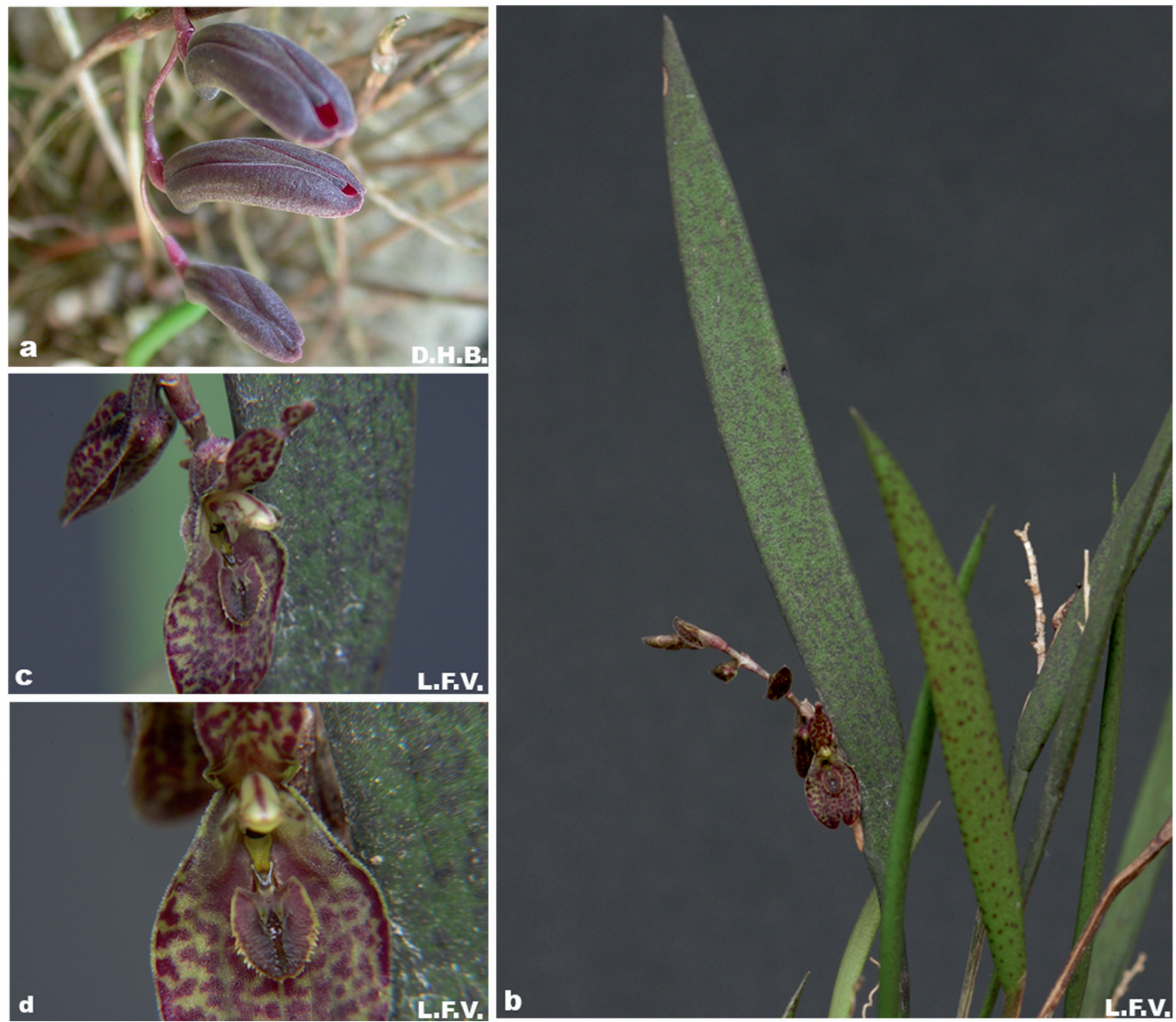

Figura 1. Acianthera sect. Pleurobotryae. a. Acianthera atropurpurea: detalhe da inflorescência. b-d. Acianthera crepiniana. b. Detalhe da inflorescência e folhas lateralmente compressas. c. Flor em vista lateral evidenciando a face externa das sépalas pubescentes e a face interna glabra. d. Detalhe da flor evidenciando o labelo com margem denticulada. Fotos. a: Dalton Holland Baptista; b-d: Luiz Filipe Varella.

Figure 1. Acianthera sect. Pleurobotryae. a. Acianthera atropurpurea: detail of the inflorescence. b-c. Acianthera crepiniana. b. Details from the inflorescence and the laterally flatened leaves. c. Flower, side view showing the pubescent external surface and the glabrous internal face. $d$. Detail of the flower showing the lip with denticulate margin. Photos. a: Dalton Holland Baptista; b-d: Luiz Filipe Varella. 
Ginostêmio ca. 4 mm compr., subcilíndrico, delgado, encurvado, glabro.

Material examinado: BRASIL. PARANÁ: Bocaiúva do Sul, 17-IX-1969, C. Zoozicki s.n. (MBM12528); Campina Grande do Sul, 18-VIII-1966, G. Hatschbach 14609 (MBM); idem, 7-IX-1966, J.M. Silva et al. 1962 (MBM); Campo Largo, 18-IX-1949, G. Hatschbach 1559 (MBM); Carneiro, 18-X-1966, G. Hatschbach 14978 (MBM); Curitiba, 5-X-1909, R. Lange 8516 (MBM); Itaperussú, 28-VII-1909, P. Dusén s.n. (MBM136709); Lapa, 10-X-2010, E.C. Smidt 994 (UPCB); Piraquara, 8-X-1946, G. Hatschbach 502 (MBM, RB); São José dos Pinhais, 12-X-1966, G. Hatschbach 14835 (MBM, UPCB); Tijucas do Sul, 21-X-1977, G. Hatschbach 40252 (MBM); Tunas do Paraná, 5-I-2006, J.M. Silva et al. 4570 (MBM); Turvo, 18-X-2009; M.G. Caxambu s.n. (HCF8342); sine loco, 5-X-1910, R. Lange 8517 (MBM). SÃo Paulo: Apiaí, 25-VIII-1939, M. Kuhlmann s.n. (SP41462); Campos do Jordão, 18-X-1923, F.C. Hoehne s.n. (SP14595); Itararé, 5-IX-2006, F. Barros s.n. (SP401805).

Acianthera crepiniana é facilmente reconhecida entre as congêneres por ser a única espécie com folhas lateralmente achatadas. Possui flores com grande variação de colorido o que, provavelmente, levou Kraenzlin (1920) a descrever Pleurothallis albopurpurea como espécie autônoma. No entanto, a análise das duplicatas do material examinado citado no protólogo da espécie não deixa dúvidas de que este nome seja sinônimo de A. crepiniana. Apesar dessa variação, é possível distinguir três padrões de colorido nas flores de $A$. crepiniana, flores castanhas com máculas vinosas que se enquadram na descrição de Cogniaux (1893-1896), flores róseas com máculas purpúreas, que se enquadram na descrição de Pleurothallis albopurpurea, e flores totalmente verdes, desprovidas de máculas, estas últimas presentes em plantas só encontradas, até o momento, no Estado do Paraná. Embora a variação de colorido não seja suficiente para delimitação de espécies autônomas, seria possível estabelecer, com base nela, três formas distintas.

A espécie ocorre na Floresta Atlântica da região Sul do Estado de Minas Gerais, extremo Nordeste e Sul do Estado de São Paulo e no Sudoeste do Estado do Paraná onde, aparentemente, é mais abundante. Espécie preferencialmente encontrada em regiões serranas em altitudes variando de 760 a $1.600 \mathrm{~m}$ (figura 5). Há relatos da ocorrência desta espécie no Estado do Rio Grande do Sul (Giehl 2012), porém nenhum material dessa região foi encontrado nos herbários consultados.

Na descrição de Pleurothallis albopurpurea, Kraenzlin (1920) não especificou a qual herbário pertenceria o holótipo da espécie. Embora as coletas de Dusén contem com duplicatas em vários herbários, normalmente assume-se que, no caso de materiais tipo, o holótipo corresponda à duplicata depositada no herbário S. No caso específico de P. albopurpurea, no entanto, há um complicador: há duas exsicatas montadas em $\mathrm{S}$ com o mesmo número de coleta Dusén 8516, correspondendo aos números de tombo S-R 4948 e S 05-6339, ambas provenientes do mesmo local de coleta, ambas igualmente bem preservadas, ambas claramente pertencentes a P. albopurpurea (= Acianthera crepiniana), e ambas indicadas como "typus". Portanto, há necessidade de propor um lectótipo para esse nome, e, para tanto, optamos pelo material S-R 4948, pois esse, inclui uma etiqueta, aparentemente manuscrita pelo próprio Fritz Kraenzlin indicando "Pleurothallis albo-purpurea Kränzl., n. sp.”.

3. Acianthera hatschbachii (Schltr.) Chiron \& van den Berg, Richardiana 12(2): 73. $2012 \equiv$ Pleurothallis hatschbachii Schltr., Repert. Spec. Nov. Regni Veg. 23: 38. 1926 三 Pleurobotryum hatschbachii (Schltr.) Hoehne, Bol. Mus. Nac. Rio de Janeiro 12(2): 28. 1936. Tipo: BRASIL. PARANÁ: próximo a Curitiba, A. Hatschbach 5 (B, destruído). Neótipo, aqui designado: BRASIL. PARANÁ: Curitiba, 11-X-1952, G. Hatschbach 2768 (MBM). Figuras 2 a, b; 3 d, e

Planta epífita, pendente, cespitosa, $25-34 \mathrm{~cm}$ alt. Rizoma cilíndrico, articulado, ca. $1,2 \times 0,3 \mathrm{~cm}$. Cauloma 11,2-21,8 $\times 0,1-0,2 \mathrm{~cm}$, ligeiramente arqueado, 3-5-articulado, parcialmente revestido por 3-5 bainhas tubulosas que se fragmentam na maturidade. Folha 9,1-15,4 × 0,2-0,3 cm, verdeescura, ereta ou subereta, cilíndrica, séssil, ápice agudo, base atenuada. Inflorescência arqueada, com 3-7 flores sucessivas, 8,5-13 cm compr.; pedúnculo 0,6-1,9 cm compr., filiforme; bráctea floral ca. $3 \mathrm{~mm}$ compr., estramínea, tubulosa, ápice obliquamente truncado. Flores verdes ou castanhas, pontilhadas de vinoso; pedicelo ca. $2 \mathrm{~mm}$ compr., filiforme; ovário ca. $2 \mathrm{~mm}$ compr., articulado ao pedicelo, cilíndrico, glabro; sépala dorsal 9-10 × 3-4 mm, carnosa, 
subereta, estreitamente elíptica, 3-nervada, glabra, ápice agudo, tenuemente reflexo; sépalas laterais coalescentes por todo o comprimento, carnosas, suberetas, estreitamente elípticas, 3-nervadas, glabras, ápice agudo, tenuemente reflexo; pétalas ca. $4 \times 1 \mathrm{~mm}$, carnosas, eretas, estreitamente espatuladas, ligeiramente assimétricas, 1-nervadas, ápice agudo; labelo longamente unguiculado, ca. $4 \times 1 \mathrm{~mm}$, verde, carnoso, subereto, unguículo ca. $2 \mathrm{~mm}$ compr., base 2-auriculada, lâmina ca. $2 \times 2 \mathrm{~mm}$, hemi-elíptica, 3-nervada, ápice arredondado, base truncada, margem inteira. Ginostêmio ca. 4 mm compr., subclaviforme, delgado, encurvado, glabro.
Material examinado: ARGENTINA. Formosa: General Manoel Belgrano, 15-X-1996, O.N. Degiani et al. 1459 (SI); idem, 15-X-1996, O. Morrone 1459 (SI). Misiones: Cainguás, 19-IX-1990, A.E. Johnson 109 (SI); Guarani, 22-VIII-1972, B. Piccinini \& R. Cirino 2296 (SI); San Pedro, 9-IV-1995, A.E. Johnson 549 (SI). BRASIL. Paraná: Curitiba, 23-IX-1935, G. Hatschbach s.n. (SP33360); idem, 5-X-1929, F.C. Hoehne s.n. (SP24367); idem, 11-X-1952, G. Hatschbach 2768 (MBM); idem, 9-X-1903, P. Dusén 2263 (R); Guarapuava, 26-IX-1968, G. Hatschbach 19856 (MBM); Ipiranga, 8-X1969, G. Hatschbach 22395 (MBM); Irati, 30-IX-1972, P. Carvalho 38

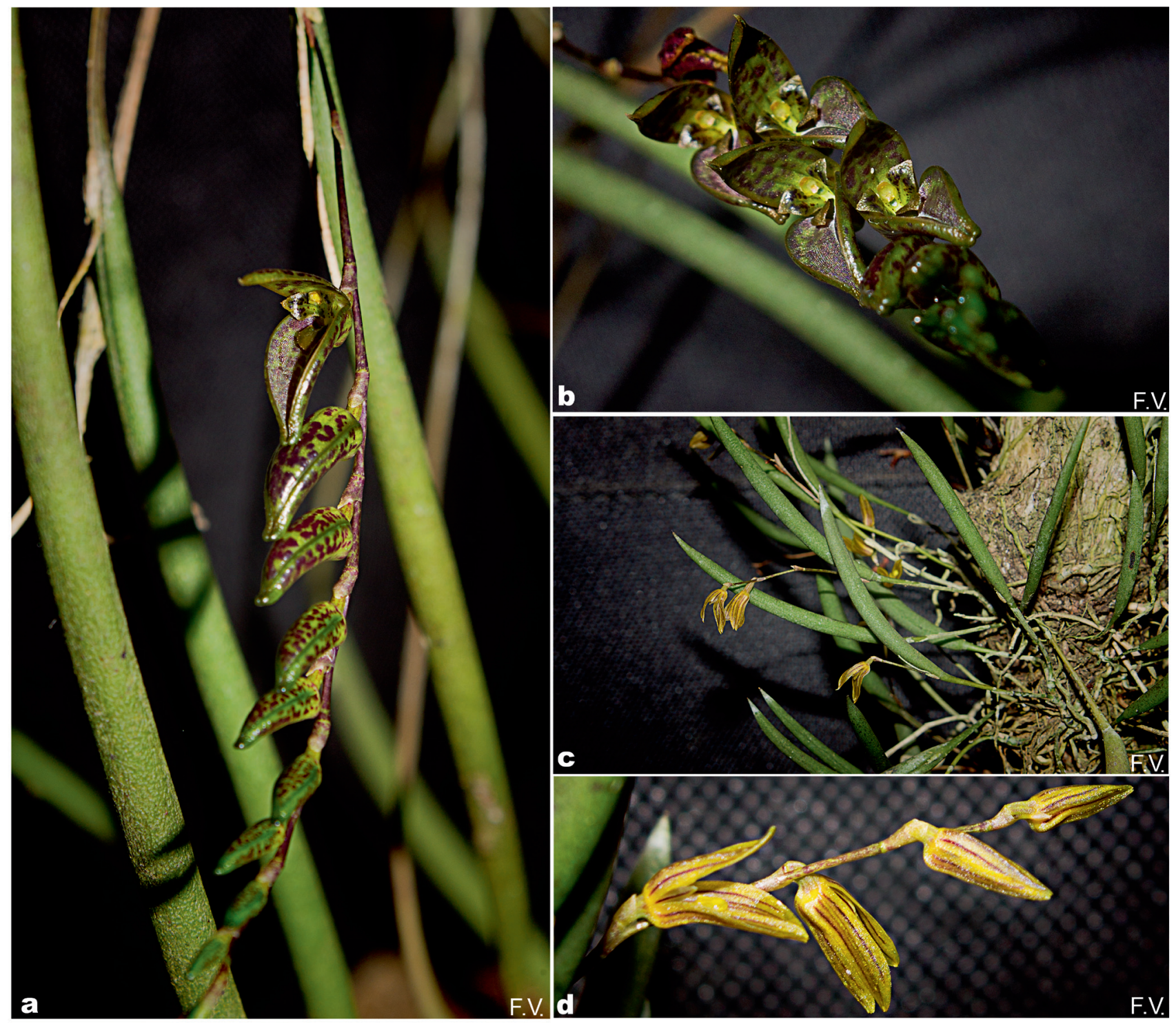

Figura 2. Acianthera sect. Pleurobotryae. a-b. Acianthera hatschbachii. a. Detalhe da inflorescência e das folhas cilíndricas. b. Vista diagonal das flores. c-d. Acianthera mantiquyrana. c. Hábito. d. Vista lateral de uma flor. Fotos: Francisney Vinhos.

Figure 2. Acianthera sect. Pleurobotryae. a-b. Acianthera hatschbachii. a. Details from the inflorescence and the cylindrical leaves. b. Oblique view of flowers. c-d. Acianthera mantiquyrana. c. General appearance. d. Lateral view of a flower. Photos: Francisney Vinhos. 


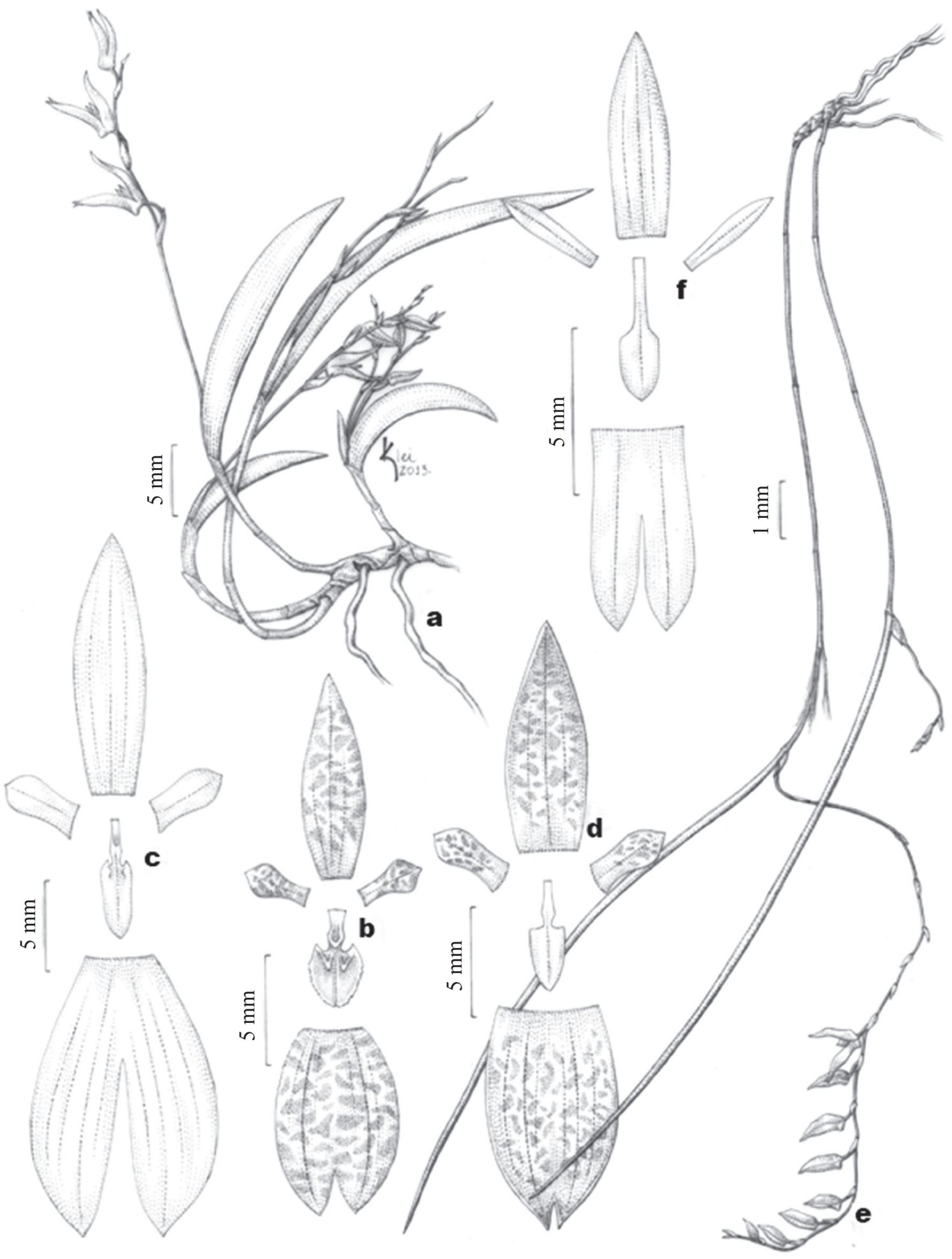

Figura 3. Acianthera sect. Pleurobotryae. a-b. Acianthera crepiniana. a. Hábito. b. Segmentos do perianto distendidos. c. Acianthera atropurpurea: segmentos do perianto distendidos. d-e. Acianthera hatschbachii. d. Segmentos do perianto distendidos. e. Hábito. f. Acianthera mantiquyrana (Barb.Rodr.) V.T.Rodrigues \& F.Barros: segmentos do perianto distendidos. Ilustração: Klei Sousa.

Figure 3. Acianthera sect. Pleurobotryae. a-b. Acianthera crepiniana. a. General appearance. b. Distended perianth parts. c. Acianthera atropurpurea: distended perianth parts. d-e. Acianthera hatschbachii. d. Distended perianth parts. e. General appearance. f. Acianthera mantiquyrana: distended perianth parts. Illustration: Klei Sousa. 
(MBM); Mangueirinha, 14-XII-1966, G. Hatschbach 15470 (MBM); Ponta Grossa, 25-IX-2006, A. Bonnet 94095 (UPCB); São João do Triunfo, 7-XI-1967, G. Hatschbach 17720 (MBM); São Mateus do Sul, 30-IX-1969, G. Hatschbach 22279 (MBM); Palmas, 10-XI-2005, J.M. Silva \& C.B. Poliquesi 4455 (MBM); Rio Negro, 23-X-1928, F.C. Hoehne s.n. (SP23150); idem, IX-1945, M. Witte s.n. (SP53082); Turvo, 27-XI-2009, M.G. Caxambu \& L. Siqueira 2812 (MBM); sine loco, 16-X-1909, R. Lange 4043 (MBM).

Acianthera hatschbachii assemelha-se a $A$. atropurpurea no hábito, mas pode ser facilmente distinguida desta por apresentar flores glabras, verdes ou acastanhadas pontilhadas de vinoso, que se abrem completamente, enquanto A. atropurpurea apresenta flores pubescentes, purpúreas, que nunca se abrem completamente. Estas espécies também podem ser distinguidas pela distribuição geográfica uma vez que A. atropurpurea ocorre nos Estados de Minas Gerais e São Paulo, enquanto A. hatschbachii ocorre no Estado do Paraná e na Argentina.

A espécie ocorre em regiões serranas e em planícies, entre 100 e $920 \mathrm{~m}$ de altitude, na Floresta Atlântica do centro-sul e sudoeste do Estado do Paraná, alcançando o extremo nordeste da Argentina, sendo encontrada, neste último país, em fragmentos de Floresta Atlântica na Província de Misiones e Formosa (figura 6).

O material originalmente utilizado como tipo por Schlechter (1926), enviado para o herbário B, não mais existe, tendo sido, provavelmente, destruído durante

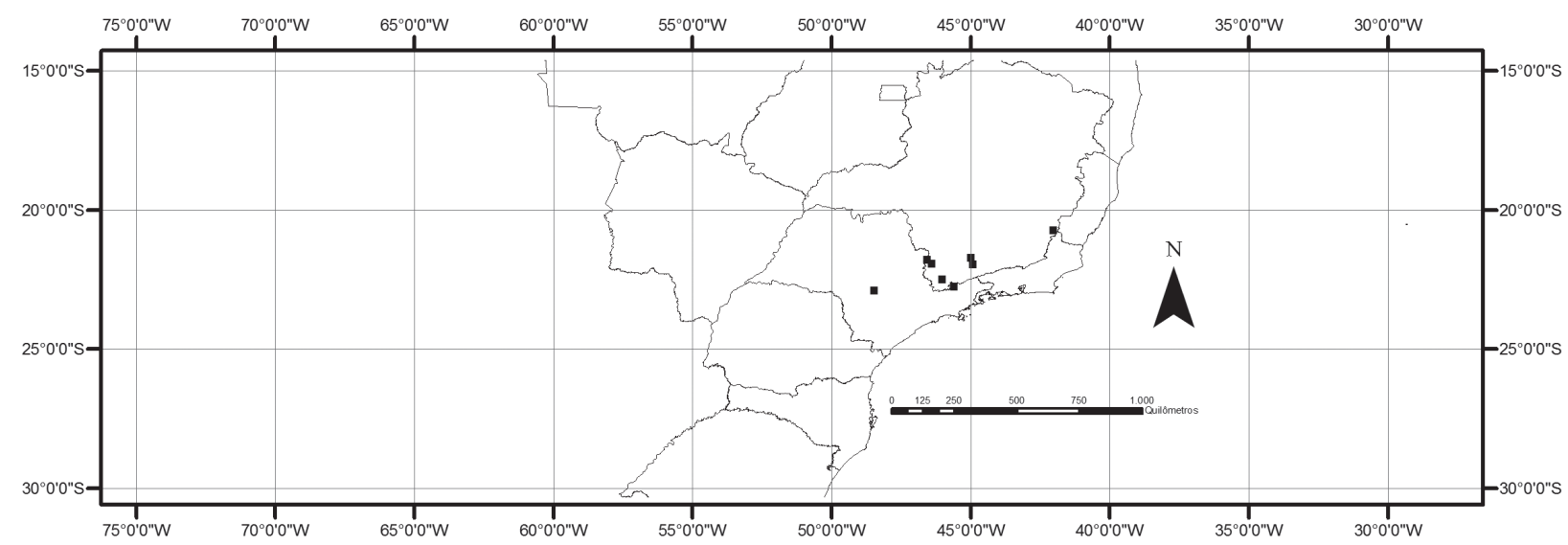

Figura 4. Distribuição geográfica de Acianthera atropurpurea, baseada no levantamento de espécies depositadas nos herbários consultados. Figure 4. Geographical distribution of Acianthera atropurpurea, based on the examined herbarium specimens.

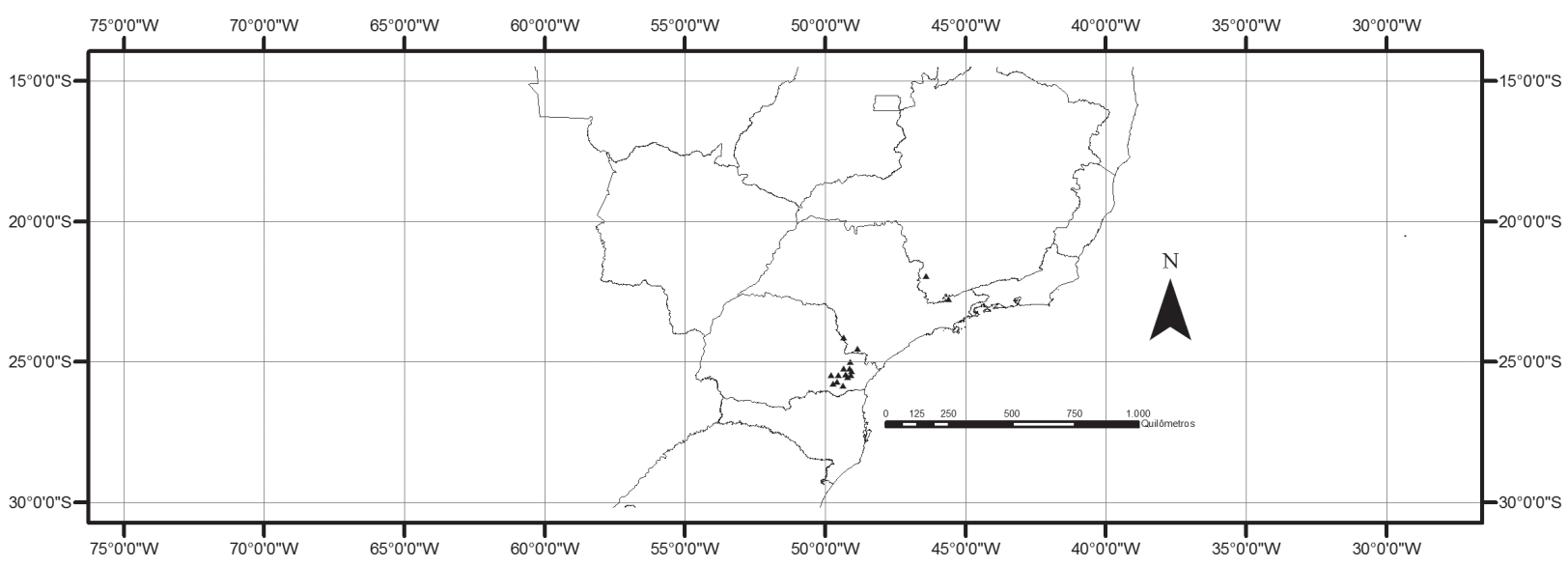

Figura 5. Distribuição geográfica de Acianthera crepiniana, baseada no levantamento de espécies depositadas nos herbários consultados.

Figure 5. Geographical distribution of Acianthera crepiniana, based on the examined herbarium specimens. 
os bombardeios a Berlin durante a Segunda Guerra Mundial; tampouco foi possível encontrar duplicatas desse material. Por esse motivo, somado ao fato de que, para essa espécie são desconhecidas ilustrações do autor, designamos a exsicata $G$. Hatschbach 2768 (MBM) como neótipo. Esse material, além de concordar bem com a descrição original, foi coletado na mesma região do tipo citado no protólogo e encontra-se em ótimo estado de conservação.
4. Acianthera mantiquyrana (Barb.Rodr.) V.T. Rodrigues \& F.Barros, comb. nov.

Basiônimo: Pleurothallis mantiquyrana Barb.Rodr., Gen. Spec. Orchid. 2: 14. $1881 \equiv$ Pleurobotryum mantiquyranum (Barb.Rodr.) Hoehne, Bol. Mus. Nac. Rio de Janeiro 12(2): 28. 1936. Tipo: BRASIL. Minas Gerais: Rio das Mortes, J. BarbosaRodrigues s.n. (holótipo RB, perdido; lectótipo aqui designado: tab.143, fig. B do v. 3 da

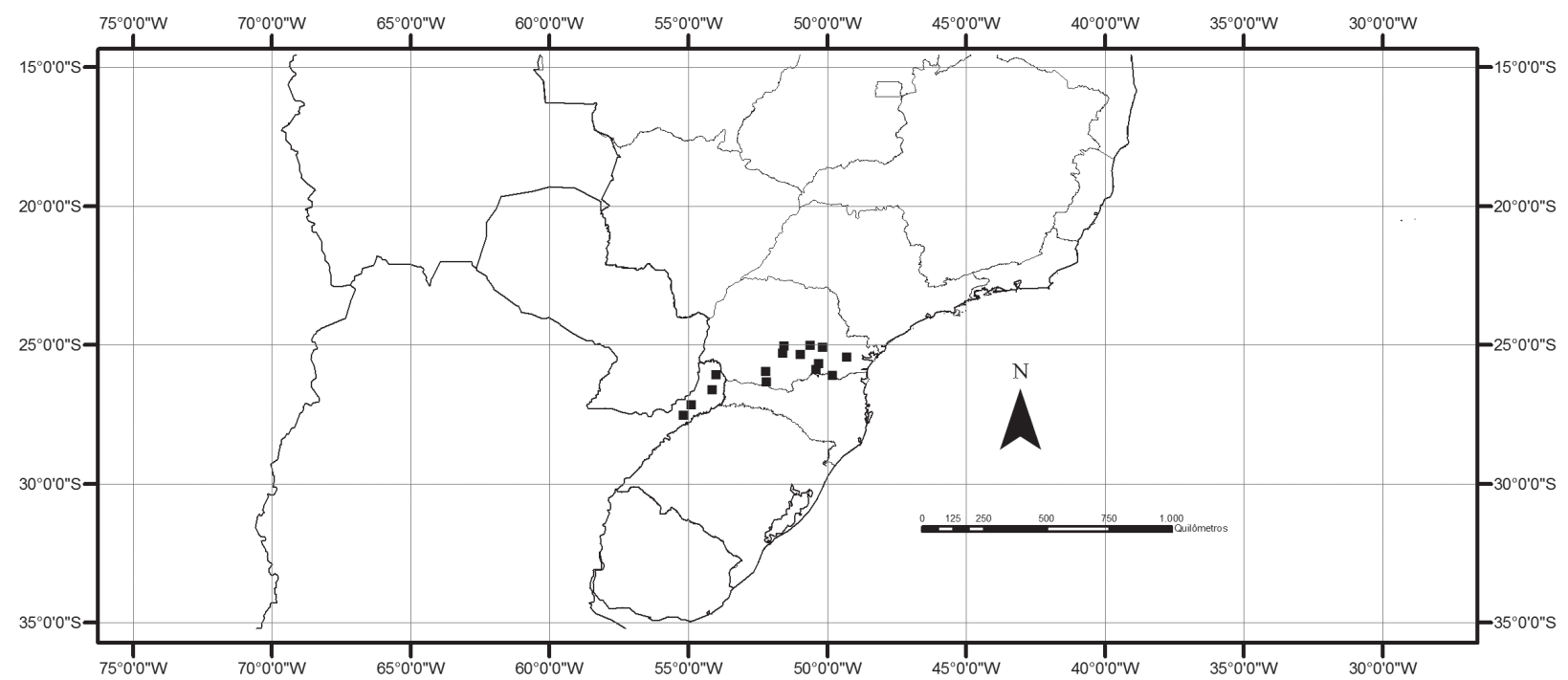

Figura 6. Distribuição geográfica de Acianthera hatschbachii, baseada no levantamento de espécies depositadas nos herbários consultados.

Figure 6. Geographical distribution of Acianthera hatschbachii, based on the examined herbarium specimens.

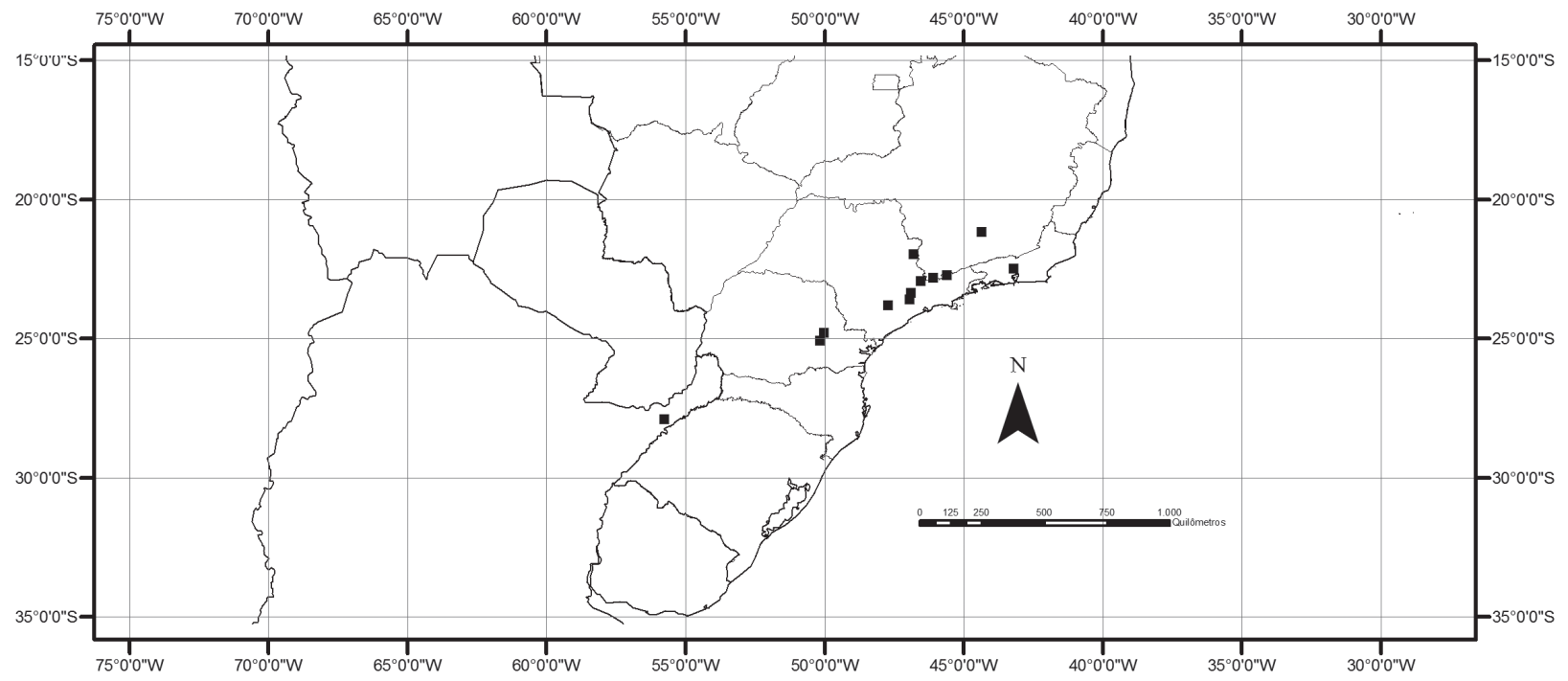

Figura 7. Distribuição geográfica de Acianthera mantiquyrana, baseada no levantamento de espécies depositadas nos herbários consultados.

Figure 7. Geographical distribution of Acianthera mantiquyrana, based on the examined herbarium specimens. 
Iconographie des Orchidées du Brésil, depositada em RB e reproduzida por Sprunger (1996, p. 201).

= Pleurothallis subulifolia Kraenzl., Orchis 2(8): 91. 1908 E Pleurobotryum subulifolium (Kraenzl.) Pabst, Orchidaceae Brasiliensis 1: 166. $1975 \equiv$ Acianthera subulifolia (Kraenzl.) Chiron \& van den Berg, Richardiana 12(2): 73. 2012. Tipo: BRASIL. Rio De Janeiro: Petrópolis, Ida Brandt s.n. (holótipo HBG501911, foto HBG!). synon. nov.

= Pleurothallis rhabdosepala Schltr., Notizbl. Bot. Gart. Berlin-Dahlem 7: 276. $1918 \equiv$ Pleurobotryum rhabdosepalum (Schltr.) Hoehne, Bol. Mus. Nac. Rio de Janeiro 12(2): 28. $1936 \equiv$ Acianthera rhabdosepala (Schltr.) Chiron \& van den Berg, Richardiana 12(2): 73. 2012. Tipo: BRASIL. Sul do Brasil. Grossmann s.n. (holótipo B, perdido; lectótipo aqui designado: tab 35, fig. Nr. 140 In: R. Mansfeld (ed.) Blüten analysen neuer Orchideen von R. Schlechter. I. Südamenikanische Or=chideen, Repertorium Specierum Novarum Regni Vegetabilis 58 (Beihefte): tab. 35, fig. 140. 1930). synon. nov.

Figuras $2 \mathrm{c}, \mathrm{d} ; 3 \mathrm{f}$

Planta epífita, ereta, cespitosa, (4-)6,5-12 cm alt. Rizoma cilíndrico, articulado, 0,8-1,2 $\times$ ca. $0,2 \mathrm{~cm}$. Cauloma (1-)2,2-5 × ca. 0,1 cm, ereto ou subereto, ligeiramente arqueado, 3-articulado, parcialmente revestido por 3 bainhas tubulosas. Folha (1,8-)3,4-5,2 × 0,1-0,2 cm, verde-escura, raro avermelhada, ereta ou subereta, cilíndrica, sulcada na nervura central, séssil, ápice agudo, base atenuada. Inflorescência ereta, 2-5 flores simultâneas; pedúnculo 1,3-2,9 cm compr., filiforme, bráctea floral ca. $2 \mathrm{~mm}$ compr., estramínea, tubulosa, ápice obliquamente truncado. Flores amarelas ou alaranjadas com estrias castanho-vinosas; pedicelo ca. $3 \mathrm{~mm}$ compr., filiforme; ovário ca. $2 \mathrm{~mm}$ compr., articulado ao pedicelo, obcônico, glabro, 6-costado; sépala dorsal 5-7 $\times$ ca. $2 \mathrm{~mm}$, membranácea, subereta, estreitamente elíptica, 3-nervada, ápice agudo, tenuemente reflexo, nervuras primárias castanho-vinosas; sépalas laterais coalescentes por $1 / 2$ do comprimento ou até por toda a extensão, membranáceas, suberetas, estreitamente elípticas, 3-nervadas, ápice agudo, tenuemente reflexo, nervuras castanho-vinosas; pétalas 3-4 × ca. $1 \mathrm{~mm}$, membranáceas, suberetas, estreitamente oblanceoladas, 1-nervadas, ápice agudo, nervuras castanho-vinosas; labelo longamente unguiculado, ca. $4 \times 1 \mathrm{~mm}$ larg., amarelado, membranáceo, ereto, unguículo ca. $2 \mathrm{~mm}$ compr., base 2-auriculada, lâmina ca. $2 \times 1 \mathrm{~mm}$, hemi-elíptica, 3-nervada, ápice arredondado, base truncada, margem inteira. Ginostêmio 3-4 mm compr., subcilíndrico, delgado, encurvado, glabro.

Material examinado: ARGENTINA. Misiones: Apóstoles, 29-XI-1943, A. Burkart 14343 (SI). Salta: Santa Victória, 2-IX-1997, A.E. Johnson 773 (SI). BRASIL. Minas Gerais: Monte Verde, 15-VIII-1963, A. Ghillany 142 (HB). PARANÁ: Castro, 29-VII-1966, G. Hatschbach 14548 (MBM, UEC, UPCB); Ponta Grossa, 28-VIII-1939, M. Kuhlmann s.n. (SP41552). SÃo Paulo: Bragança Paulista, 18-VII-1910, A.P. Duarte s.n. (SP22495); Campos do Jordão, 14-IX-1923, F.C. Hoehne s.n. (SP8670); Cotia, 9-IX-1926, F.C. Hoehne s.n. (SP17273); São João da Boa Vista, s.d., A. Loefgren CGGSP2600 (SP); São Paulo, 28-XIII-1921, A. Gehrt s.n. (SP5774); idem, 1-IX-1937, O. Handro s.n. (SP47571), Pilar, 24-VIII-1939, M. Kuhlmann s.n. (SP41510).

Acianthera mantiquyrana é facilmente caracterizada entre as demais espécies da seção por ser, entre as espécies com folhas cilíndricas, uma planta comparativamente menor e ereta, com flores amarelas e estriadas de castanho-vinoso.

Ao descrever Pleurothallis subulifolia, Kraenzlin (1908) comparou-a com P. teretifolia Rolfe e P. atropurpurea (Barb.Rodr.) Cogn., fazendo alusão ao labelo longamente unguiculado, característica comum entre as três. Além das características morfológicas o autor distinguiu $P$. subulifolia por sua distribuição geográfica, afirmando que esta ocorreria em Petrópolis (Rio de Janeiro) enquanto as demais, nos Estados de Pernambuco e Minas Gerais. A análise da fotografia do holótipo [Ida Brandt s.n. (HBG501911)] e da descrição taxonômica original, indicam que este nome é sinônimo de Acianthera mantiquyrana. Ambas possuem flores morfologicamente semelhantes tanto no tamanho e colorido quanto na forma dos segmentos do perianto. Acianthera mantiquyrana é a espécie de maior distribuição geográfica na seção Pleurobotryae, ocorrendo preferencialmente em áreas a 500-2.000 m de altitude, não sendo incomum, portanto, que seja encontrada na região serrana do Estado do Rio de Janeiro como afirmado por Kraenzlin (1908) para P. subulifolia. É importante notar que, embora Kraenzlin (1908) não utilize a palavra "holotypus" ao indicar a coleta Ida Brandt s.n. como typus de Pleurothallis subulifolia, a exsicata depositada no herbário HBG (núm. 501911) possui a designação de "typus", em uma etiqueta grafada com a letra do próprio Fritz Kraenzlin, 
e de "holotypus" na etiqueta que indica a origem da exsicata, proveniente do herbário do mesmo autor ("Ex Herb. Fr. Kränzlin", na etiqueta).

Hoehne (1929) comentou que Pleurothallis rhabdosepala Schltr. parece ser apenas uma forma de locais mais expostos ao sol, enquanto $P$. mantiquyrana prefere áreas de mata sombria. Schlechter (1918) distinguiu $P$. rhabdosepala de $P$. mantiquyrana pelo tamanho das plantas, pois a primeira seria bem menor (até $7 \mathrm{~cm}$ alt.). As características florais são frágeis para delimitação de $P$. rhabdosepala como espécie autônoma, assim como a distribuição geográfica, pois embora de acordo com Schlechter (1918), P. rhabdosepala seria endêmica da região Sul do Brasil, plantas com o mesmo porte são encontradas em regiões da Serra da Mantiqueira, em Campos do Jordão (SP) e no sul do Estado de Minas Gerais, área de ocorrência de P. mantiquyrana. Os materiais analisados provenientes da região Sul do Brasil frequentemente excedem o tamanho indicado por Schlechter (1918), o que parece indicar que a espécie apresenta variação no tamanho das plantas em resposta a pressões ambientais, o que impossibilita o uso do tamanho como base para separação dessas duas espécies.

Dentre as espécies de Acianthera sect. Pleurobotryae, esta é a que apresenta distribuição geográfica mais ampla, ocorrendo na Floresta Atlântica no Sudoeste do Estado do Rio de Janeiro, Sudeste e Sul do Estado de Minas Gerais, Nordeste, Leste e Sudoeste do Estado de São Paulo, Leste do Estado do Paraná e extremo Nordeste da Argentina. Há relatos da ocorrência desta espécie no município de Santa Maria no Estado do Rio Grande do Sul (Giehl 2012), porém nenhum material de herbário foi encontrado para esta localidade (figura7).

\section{Literatura citada}

Barbosa-Rodrigues, J. 1877. Genera et Species Orchidearum Novarum, v. 1. C. et H. Fleiuss, Rio de Janeiro.

Barros, F., Rodrigues, V.T. \& Batista, J.A.N. 2009. Orchidaceae. In: J.R. Stehmann, R.C. Forzza, A. Salino, M. Sobral, D.P. Costa \& L.H.Y. Kamino (orgs.). Plantas da Floresta Atlântica. Instituto de Pesquisa Jardim Botânico do Rio de Janeiro, Rio de Janeiro, pp. 372-403.

Barros, F., Vinhos, F., Rodrigues, V.T., Barberena, F.F.V.A., Fraga, C.N., Pessoa, E.M., Forster, W., Menini Neto, L., Furtado, S.G., Nardy, C., Azevedo, C.O. \& Guimarães, L.R.S. 2015. Orchidaceae. In: R.C. Forzza et al. (orgs.). Lista de espécies da flora do Brasil. Jardim Botânico do Rio de Janeiro, Rio de Janeiro. Disponível em http://floradobrasil.jbrj.gov.br/ jabot/floradobrasil/FB10986 (acesso em 24-III-2015).
Brade, A.C. \& Pabst, G.F.J. 1952. Index generum et specierum Orchidacearum Brasiliensium inter MCMXXXII et MCML descripta sunt. Orquídea (Rio de Janeiro) 14: 70-76.

Chiron, G.R. \& van den Berg, C. 2012. Révision taxonomique du genre Acianthera (Orchidaceae, Pleurothallidinae). Richardiana 12: 59-77.

Chiron, G.R., Guiard, J. \& van den Berg, C. 2012. Phylogenetic relations in Brazilian Pleurothallis sensu lato (Pleurothallidinae, Orchidaceae): evidence from nuclear ITS rDNA sequences. Phytotaxa 46: 34-58.

Correa, M.N. 1975. Notas orquidológicas II. Cuatro géneros de Orchidaceae nuevos para la Flora Argentina. Darwiniana 19: 345-356.

Cogniaux, A. 1893-1896. Orchidaceae. In: C.F.P. Martius, A.G. Eichler \& I. Urban (eds.). Flora Brasiliensis. Fried. Fleischer, Monachii, v. 3, pars 4, pp. 1-672.

Dressler, R.L. 1981.The Orchids - Natural history and classification. Harvard University Press, Cambridge.

Garay, L.A. 1952. Notatio Orchidologica II. Archivos do Jardim Botânico do Rio de Janeiro 12: 169-186 + pl. 1-4.

Giehl, E.L.M. 2012. Flora digital do Rio Grande do Sul e de Santa Catarina. Disponível em http://www.ufrgs.br/ fitoecologia/florars/ (acesso em 30-03-2013).

Hoehne, F.C. 1929. Contribuição para a flora orquidológica brasílica. Archivos do Instituto Biologico de Defesa Agrícola e Animal 2: 5-52.

Hoehne, F.C. 1936. Orchidaceas dos Herbários de Alexandre Curt Brade e do Museu Nacional. Boletim do Museu Nacional 12: 1-37.

Kraenzlin, F. 1908. Neue und kritische Arten. Orchis 7: 89-92.

Kraenzlin, F. 1920. Orchidaceae Dusenianae Novae. Arkiv för Botanik 16: 1-30.

Lindley, J. 1842. Pleurothallis. Edwards's Botanical Register (Miscellaneous matter) 28: 67-84.

Luer, C.A. 1986. Icones Pleurothallidinarum I. Systematics of the Pleurothallidinae (Orchidaceae). Monographs in Systematic Botany from the Missouri Botanical Garden, v. 15. Missouri Botanical Garden, Saint Louis.

Luer, C.A. 2004. Icones Pleurothallidinarum XXVI. Pleurothallis subgenus Acianthera and three allied subgenera. A second century of new species of Stelis of Ecuador. Epibator, Ophidion, Zootrophion. Monographs in Systematic Botany from the Missouri Botanical Garden, v. 95. Missouri Botanical Garden, Saint Louis.

Luer, C.A. 2006. Icones Pleurothallidinarum XXVIII. A reconsideration of Masdevallia. Systematics of Specklinia and vegetatively similar taxa (Orchidaceae). Monographs in Systematic Botany from the Missouri Botanical Garden, v. 105. Missouri Botanical Garden, Saint Louis.

Pabst, G.F.J. \& Dungs, F. 1975. Orchidaceae Brasilienses v. 1. Kurt Schmersow, Hildesheim. 
Pridgeon, A.M. \& Chase, M.W. 2001. A phylogenetic reclassification of the Pleurothallidinae (Orchidaceae). Lindleyana 16: 235-271.

Pridgeon, A.M., Solano, R. \& Chase, M.W. 2001. Phylogenetic relationships in Pleurothallidinae (Orchidaceae): combined evidence from nuclear and plastid DNA sequences. American Journal of Botany 88: 2286-2308.

Pridgeon, A.M., Cribb, J.P., Chase, M.W. \& Rasmussen, F.N. 2005. Genera Orchidacearum. v. 4. Epidendroideae (Part one). Oxford University Press, Oxford.

Radford, E.A., Dickison, W.C., Massey, J.R. \& Bell, C. 1974. Vascular plant systematics. Haper \& Row, New York.

Rolfe, R.A. 1892. New and noteworthy plants. The Gardener's Chronicle, series 3, 12: 521.
Schlechter, R. 1918. Orchidaceae novae, in caldaris Horti Dahlemensis cultae. Notizblatt des Botanischen Gartens und Museums zu Berlin-Dahlem 7: 268-280.

Schlechter, R. 1926. Beiträge zur Kenntnis der Orchidaceen flora von Parana II. Orchidaceae Hatschbachianae. Repertorium Specierum Novarum Regni Vegetabilis 23: 32-71.

Sprunger, S. (ed.). 1996. João Barbosa Rodrigues Iconographie des orchidées du Brésil. v. 1: The illustrations. Friedrich Reinhardt, Basle.

Thiers, B. 2013 [continuously updated]. Index Herbariorum: A global directory of public herbaria and associated staff. New York Botanical Garden's Virtual Herbarium. Disponível em http://sweetgum.nybg.org/ih/ (acesso em 25-III-2013). 